\title{
Generation Scheduling in Power Systems with Hydro Electric Plants
}

\author{
S. Palamarchuk ${ }^{*}$
}

\author{
Energy Systems Institute, 130 Lermontov St., Irkutsk 664033, Russia
}

\begin{abstract}
Medium-term generation scheduling is an important component of power systems operation and management. The traditional problem statement aimed at reducing the total production cost can hardly correspond to the market environment. The paper considers specific features of the problem statement for a wholesale electricity market environment. An approach is suggested to solve the problem on the basis of bi-level optimization models. Such models take into account possible distortion of economic and technical parameters of generating units. The proposed technique obtains equilibrium of the generation company's interests to simulate the competitive behavior under the oligopoly electricity market. A mathematical statement of the problem supposes the application of a dynamic programming method. An algorithm for the stochastic dynamic programming application is developed. A numerical example is presented to demonstrate the applicability of the method and algorithm. The efficiency of the proposed approach is shown in comparison with the traditional generation scheduling technique.
\end{abstract}

Keywords: Power system management; medium-term scheduling; market environment; bi-level approach; dynamic programming.

\section{INTRODUCTION}

Generation scheduling is a traditional problem solved to operate and manage Electric Power System (EPS). The problem is particularly important for EPS with a large contribution of hydroelectric power plants (HPPs) with significant water reservoirs. Hydropower utilization is currently considered as an important aspect in the development of cleaner and more efficient energy. System Operator (SO) of EPS schedules electricity generation for a short- or medium-term perspective. The medium-term scheduling supposes consideration of the EPS operation through a period from several weeks up to one year. The scheduling period is divided into several time intervals. Duration of the intervals can be different depending on "variability" of power system state and certainty of input information to be applied. Operation conditions and load flow variables within each interval are taken as invariable. Forecasted information on electricity consumption, network configuration and cost functions of generating units is considered as given data.

Scheduling of the HPP outputs requires that account be taken of water reserves and levels in reservoirs, water flow rate through turbines and draw downs, forecast of lateral inflow to reservoirs and cascade location of plants along the water stream. Electricity generation at HPP is largely determined by water utilization and environmental constraints. The constraints can restrict the EPS operation in some time intervals.

*Address correspondence to this author at the Energy Systems Institute, 130 Lermontov St., Irkutsk 664033, Russia; Tel: 78950131 0100; Fax: 7935242 6796; E-mail: palam@isem.sei.irk.ru
Scheduling the operation of EPSs that involve HPPs requires consideration of the interdependence of decisions to be made in certain time intervals. The water volumes for electricity generation at a specific HPP in some time intervals affect loading of this plant during the remaining time of the scheduled period. Besides, the decision on the use of water in certain time intervals affects the total output of the plants of different type and the total economic efficiency of the EPS operation.

Initial information employed in solving the problem is highly uncertain. Mainly, it concerns the information about electricity demand and particularly about water inflow to the reservoirs. For time intervals such information is normally represented in a probabilistic form.

Mathematically, the generation scheduling is represented by a multi-interval optimization problem of stochastic dynamic programming with constraints associated with several time intervals.

Results of generation scheduling are needed by generation companies (GenCos), electricity consumers and market regulators. Generation companies provide power plants with a certain amount of fuel, consumers develop their strategies of participation in the electricity markets and regulators establish transmission and distribution prices.

Traditionally, the generation scheduling problem has aimed to attain the minimum of variable part (fuel component) of the total production cost at thermal power plants (TPPs) in the EPS. In the last decade 
electric power industry in many countries has undergone radical changes. Interaction of vertically integrated energy companies is replaced with competitive liberalized electricity markets. The traditional statement of the problem can hardly correspond to the market environment. New factors should be taken into account. These are:

GenCos prepare the cost functions of their generating units and submit them to SO. GenCos are able to distort parameters of the cost functions to increase their profit;

- Power plants are integrated into GenCos that pursue their corporate aims and seek to maximize the company profit. Each GenCo can include several power plants located at different nodes in EPS. Generation scheduling should consider the operation of a power plant as part of GenCo rather than an independent supplier;

In some countries the criterion of decision making in operation scheduling was changed. The traditional criterion of the "minimum total electricity production costs in the EPS" was replaced by the "minimum purchase costs for electricity buyers";

Some GenCos can affect market prices by changing the volumes of outputs and manipulating parameters of cost functions. Electricity markets are the markets with imperfect competition. The scheduling problem should take into account the oligopoly situation in the market;

Electricity demand became sensitive to the market price deviations in a medium-term perspective.

The market transformations in the power sectors influence the practice of generation scheduling. The necessity arises to adjust the normative documents and scheduling procedures.

Nowadays there are numerous studies on the problem of generation scheduling of hydrothermal systems in the competitive market environment. The researches done by C. Carraretto [1], J. Bushnell [2], M.V. Pereira [3 4], H. Rudnick [5] and others are aimed at developing models of hydrothermal systems for profit maximization of every generating company. An approach devoted to bi-level optimization problems and equilibrium investigation has appeared in the papers by
B.F. Hobbs [6], A. J. Conejo [7], J. D. Weber, T. J. Overbye [8], J. Barquin [9] and others. Nevertheless, the mathematical formulation of the problem, methods and algorithms for solving it are not developed thoroughly enough.

The paper presents a bi-level statement of the problem and algorithm for medium-term generation scheduling, taking account of EPS operation in the wholesale market environment. Only one commodity, i.e. electricity, is considered to be traded. The other kinds of markets (for auxiliary services and capacity) are not considered in the paper.

\section{A BI-LEVEL APPROACH TO GENERATION SCHEDULING}

Let the production cost function of producer $i$ at time interval $t$ be

$C_{i}^{t}\left(P_{g i}^{t}\right)=a_{i}^{t}+b_{i}^{t} P_{g i}^{t}+0.5 c_{i}^{t}\left(P_{g i}^{t}\right)^{2}$

where $P_{g i}^{t}$ is power generation at bus $i$ and time interval $t$.

To schedule electricity generation under the competitive market SO receives economic characteristics of generating equipment from individual suppliers. Besides, the suppliers submit the information on availability of their generating capacities $P_{g i \max }^{t}$ in certain time intervals. SO schedules outputs on the assumption that the submitted characteristics and $P_{g i \max }^{t}$ reflect the true production costs and capacity capabilities of power plants.

Unfortunately, in the market environment such assumptions are not always true. Suppliers can distort parameters of the submitted characteristics and capacity limits in order to increase their profit. The possibility of such manipulations is explained by imperfect competition in the electricity markets. Some GenCos with TTPs can simultaneously submit the distorted parameters $\bar{b}_{i}^{t}, \bar{c}_{i}^{t}$ of costs characteristics and distorted output limits $\bar{P}_{g i \max }^{t}$ of power plants. Some suppliers can specify only distorted limits.

The scheduling problem is solved at two levels. At the first (lower) level the strategic suppliers prepare technical and economic data on their generating units to submit them to SO. At the second (upper) level SO determines loading of generating and network equipment in certain time intervals of the scheduled period. 
The problem of the first level is solved by each strategic supplier (GenCo) bearing in mind the expected behavior of other suppliers and also taking account of the fact that the prepared information will be then used by SO to schedule the power plant outputs. Behavior of suppliers depends on the conditions emerging in the wholesale market (price levels, outputs of other GenCos, constraints on power transmission, deliveries stipulated by bilateral contracts). To maximize the profit the supplier $f$ should predict electricity prices at EPS nodes. The expected data on behavior of other suppliers are external parameters for supplier $f$ at the first level of problem solving.

The initial data for the first level sub-problem are nodal prices $p_{i}^{t} \quad\left(i \in I_{n}\right), P_{g i}^{t}\left(i \in I_{g}\right)$, production cost and consumer demand functions. The variables of the first level sub-problem are $\bar{b}_{i}^{t}, \bar{c}_{i}$ and $\bar{P}_{\text {gimax }}^{t}, t \in T$ for all power plants which belong to the strategic GenCo. Constraints on the values of distorted parameters $\bar{b}_{i}^{t}$, $\bar{c}_{i}$ and limits $\bar{P}_{\text {gimax }}^{t}$ for power generation can be introduced into this sub-problem.

The first level sub-problem for strategic GenCo $f$ at interval $t$ is represented as

$$
\begin{aligned}
& \max _{\bar{b}_{i}^{t}, \bar{c}_{i}^{t}, \bar{P}_{\text {giax }}^{t}} S_{f}^{t}=\sum_{i \in I_{f}}\left(P_{g i}^{t} \cdot p^{t}{ }_{i}-C_{i}^{t}\left(P_{g i}^{t}\right)\right)+E S_{f}^{t+1, T}\left(\tilde{P}_{g i}^{t+1, T}\right), \\
& \bar{b}_{i \min }^{t} \leq \bar{b}_{i}^{t} \leq \bar{b}_{i \max }^{t}, i \in I_{f}, \\
& \bar{c}_{i \min }^{t} \leq \bar{c}_{i}^{t} \leq \bar{c}_{i \max }^{t}, i \in I_{f}, \\
& \bar{P}_{\text {gimin }}^{t} \leq \bar{P}_{\text {gimax }}^{t} \leq P_{g i \max }^{t}, i \in I_{f}
\end{aligned}
$$

In (1)-(4) $\bar{b}_{i \text { min }}^{t}, \bar{c}_{i \text { min }}^{t}$ and $\stackrel{-t}{b}_{i \max }, \stackrel{-t}{c}_{i \text { max }}$ are minimal and maximal limits for distorted parameters $\bar{b}_{i}^{t}$ and $\bar{c}_{i}^{-t}$; $\bar{P}_{g i \min }^{t}$ is a minimal limit for distorted generation capacity $\bar{P}_{g i \max }^{t}, M W ; E S_{f}^{t+1, T}$ is the expectation of the future profit of GenCo $f$ from interval $t+1$ to the end of the scheduling period; $I_{f}$ is set of generating buses in the $f$-th GenCo; $T$ is a set of time intervals.

The sub-problem of the second level is reduced to the centralized generation scheduling and achievement of the minimum costs of electricity purchase for consumers. At the second level SO applies the data prepared and submitted by suppliers at the first level. It obtains amounts of power outputs in the generation buses and power flows in the network, as well as the levels of nodal prices. Technical constraints on power balance in network buses, range of generating capacities and transfer capabilities of network ties are considered together with hydrological constraints at the second level.

The decisions made by suppliers at the first level affect load flow variables determined by $\mathrm{SO}$ at the second level. In turn, decisions made by $\mathrm{SO}$ at the second level influence the decisions of suppliers that are made at the first level. Modeling of such interrelations is based on bi-level optimization problems with different kinds of constraints [10]. Such problems are used to study the properties of electricity markets with imperfect competition $[7,11,12]$.

The nodal price determination and subsequent actions of SO are modeled within the first level subproblem by the Kuhn-Tucker optimality conditions corresponding to the sub-problem of the second level. The sets of constraints for the first and second level sub-problems are described in $[13,14]$.

\section{INTEREST EQUILIBRIUM OF GENERATION COMPANIES}

Each strategic GenCo in the market environment seeks to increase its profit and solves the first level sub-problem (1)-(4). Thus, the sub-problem should be considered as a multi-objective optimization problem. Interdependence of the decisions made by individual GenCos is the basic feature of the electricity markets with imperfect competition. Every decision to be made by strategic GenCo should take into consideration possible response from competitors $[5,7,9]$.

The following assumptions are introduced for generation scheduling in the oligopoly market environment:

Each GenCo tends to increase its profit from electricity sale in the wholesale market;

Strategic GenCos simultaneously prepare and submit their data to SO;

When making decisions GenCos have no precise information about the output capabilities and cost functions of competitors. GenCos apply 
the estimated data on parameters and behavior of competitors while solving the first level problem;

- $\quad$ Strategic GenCos enjoy equal rights. There is no leading company with a privilege of submitting their information;

- $\quad$ The cartel collusion among strategic suppliers is not considered;

The first level sub-problem in the market with imperfect competition can be considered as a Nash game with several players. Each GenCo solves not an ordinary but a bi-level optimization problem with calculation of nodal prices for consumers and suppliers. The final problem objective is to find an equilibrium at which in time intervals the suppliers have no incentive to unilaterally change economic characteristics of their equipment and electricity generation.

The first level sub-problem (1)-(4) solved at each time interval is a non-linear optimization problem with non-linear $C_{i}^{t}\left(P_{g i}^{t}\right)$ and $S_{f}^{t+1, T}\left(P_{g i}^{t+1, T}\right)$ functions. The determination of the equilibrium conditions for the nonlinear problems is not a trivial task. In the general case there can be absence or multiplicity of equilibriums. Investigation of the equilibrium properties is not the aim of this paper. The detailed mathematical analysis of problem (1)-(4) will be made to study the equilibrium convergence later.

There are two approaches to the determination of the equilibrium solution at a time interval.

\section{Problem Solving with Application of the Mixed Complementarity Method}

The idea of the approach is to substitute every bilevel optimization problem by its Kuhn-Tucker optimality conditions (complementarity problem). All optimality conditions are combined into an integrated system of equations and inequalities (mixed complementarity task). Solution to the integrated system provides strategic suppliers with the equilibrium generation schedule $[11,15]$.

The method requires that very large systems of equations be set up and solved. Application of the Mixed Complementarity method for generation scheduling in real EPS with a complex electric network and multi-interval scheduling period necessitates significant computational efforts.

\section{Problem Solving with Iterative Search for the Equilibrium State}

The calculation starts with the application of cost functions specified in advance for all generating nodes. In each iteration supplier $f$ maximizes its profit on the assumption that the cost functions and competitors' output capabilities are known and fixed. Supplier $f$ determines the parameters $\bar{b}_{i}, \bar{c}_{i}, i \in I_{f}$ of cost functions and the values of limits $\bar{P}_{g i \max }, i \in I_{f}$ for power plants in its GenCo. Simulation of SO's actions allows the expected outputs of power plants belonging to other GenCos in EPS to be calculated.

In the next iteration the following supplier $f+1$ takes the data of competitors as known and solves a bi-level problem. The iterations continue, until it turns out to be profitable for all suppliers not to change the parameters of cost functions and the output limits of their power plants [9, 12]. The obtained equilibrium solution is submitted to SO for generation scheduling at the second level.

Generation scheduling in the power systems is carried out regularly season by season and year by year. Therefore, the scheduling problem may be treated as a repeated dynamic game with changing game parameters. The existence of equilibriums should be investigated for such a game. However, the repeated character of the scheduling is not taken into account in this paper. Generation scheduling is considered here for one independent scheduling period.

\section{METHOD AND ALGORITHM FOR GENERATION SCHEDULING}

A dynamic programming method is used for solving the medium-term scheduling problem. The main ideas of the method and algorithm were developed in [4] with calculations in terms of the backward induction. The following modifications were proposed and added to the algorithm in our study:

- $\quad$ simplified modeling of transmission losses;

- decomposition of the scheduling problem into the first and the second level sub-problems;

- consideration of the first level sub-problem as a multi-objective task;

- $\quad$ attainment of the interest equilibrium of GenCos treating the first level sub-problem as a Nash game. 
The essence of the proposed method lies in the decomposition of the problem into two stages: direct and inverse. The number of variables at the beginning and the end of the scheduling period are assumed to be known. The steps of the algorithm for inverse and direct stages are shown in Figures $\mathbf{1}$ and $\mathbf{2}^{1}$. Calculations start from the inverse stage. The last interval of the scheduling period is considered.

It is supposed that at the beginning of each interval $t$ several volumes of water $V_{j l}^{t}$ accumulated in reservoir of $j$-th HPP are given ( $/$ is the index of water volume; $L$ is number of given volume values). For each $V_{j l}^{t}$ several scenarios of random lateral water inflow $\tilde{A}_{j l m}^{t}$ (step 3) are set with the assumed probability ( $m$ is index of scenario, $M$ is number of scenarios). The scenarios are determined by the long-term hydrological forecasting.

Whereas the costs of electricity generation at hydro power plants are usually less than production costs at TPPs, HPPs should be loaded first to gain the best economic effect. At step 4 the maximum generation of HPPs is determined taking into account technical and hydrological constraints.

The bi-level problem for GenCos is solved at step 5 of the algorithm (Figure 1). The future profit $E S_{f}^{t+1, T}$ is equal to zero in expression (1) for the last time interval. The Mixed Complementarity method is used to attain equilibrium at this step. The Kuhn-Tucker optimality conditions replacing the first level sub-problems of other suppliers are applied in addition to constraints (2)-(4). Solving the mixed complementarity problem we obtain the total production costs for a certain lateral water inflow scenario and for certain water volume in reservoirs. Similar calculations are carried out for other lateral water inflow scenarios (steps 3-6) and consequent time intervals. After the probabilistic assessment of the results, the points of Future Profit Functions (FPFs) are obtained for each GenCo (step $8)$. Then, the calculations are made for the other water storage volumes (steps 2-9).

FPFs for the considered time interval are plotted using the obtained points (step 11) and are approximated by the analytical expressions through any known procedure. The FPFs for the remaining time intervals are plotted similarly (steps 1-12). The inverse

\footnotetext{
${ }^{1}$ The algorithm and computer program for generation scheduling are developed by post graduate student I.A. Nechaev.
}

stage is considered to be solved when FPFs are obtained for all time intervals of the scheduling period.

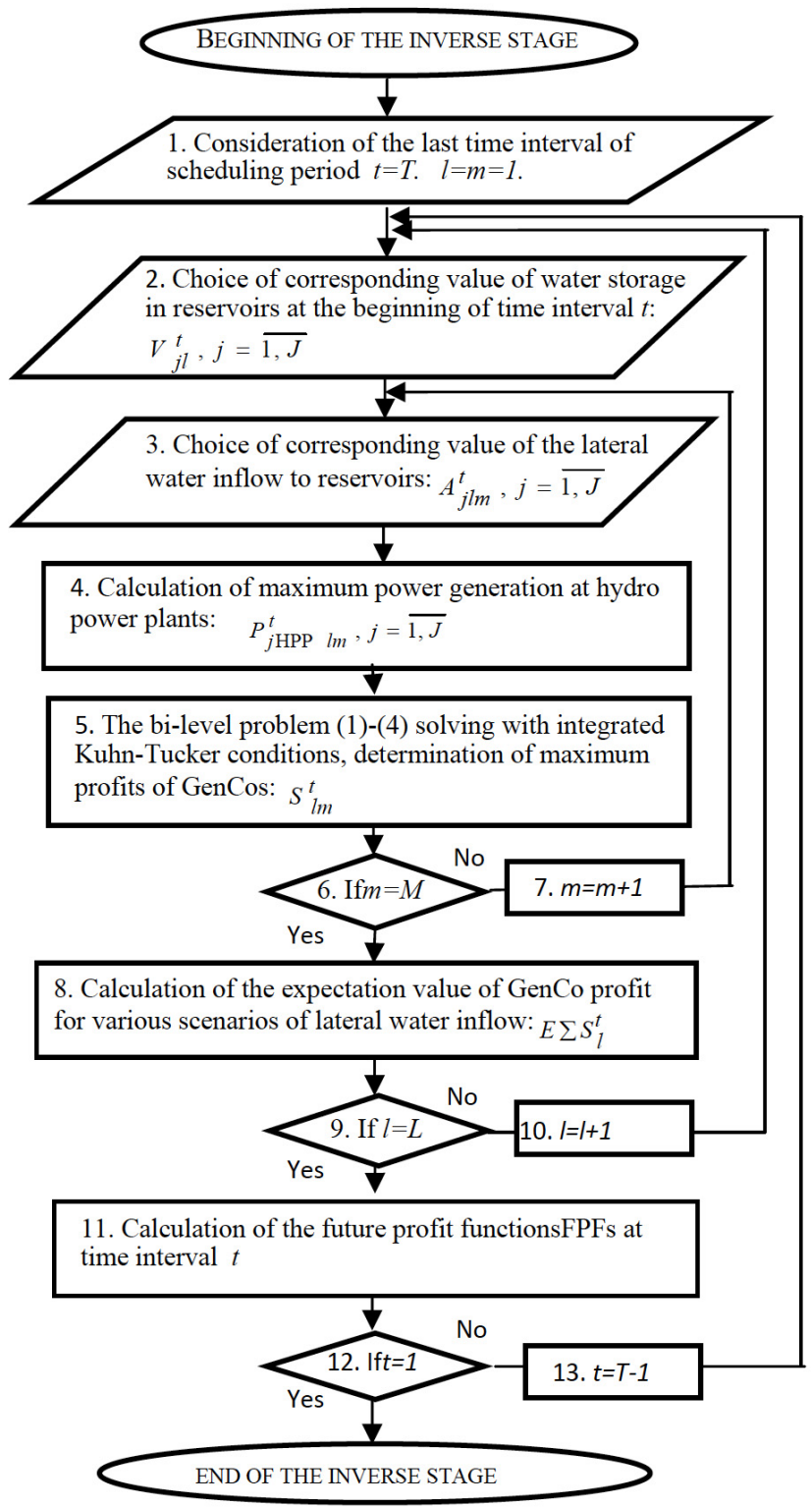

Figure 1: The control flow chart of the inverse stage of calculations.

Consideration of the direct stage begins with the first time interval (Figure 2). In the objective function we use FPFs plotted at the inverse stage. Constraints on hydrological and technical variables are taken into account as well. As a result the optimal outputs of power plants at each time interval are obtained, and hence the problem is solved.

\section{CASE STUDY}

Efficiency of the offered technique and algorithm is tested by a numerical example. Factors affecting the 


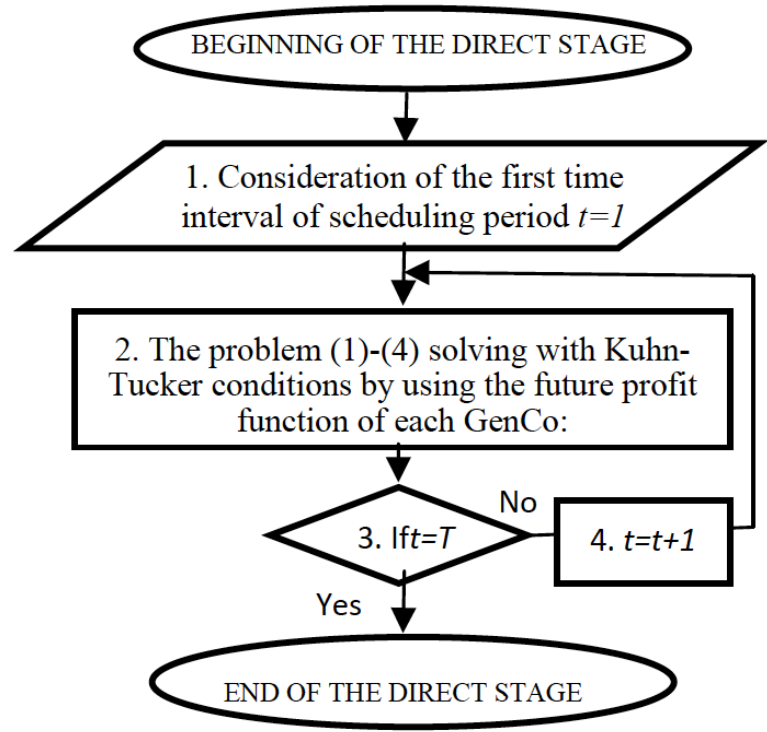

Figure 2: The control flow chart of the direct stage of calculations.

cost structure and profit of GenCos are analyzed for the medium-term generation scheduling. One section of the Russian electric power grid has been chosen as a test system (Figure 3 ). The test system contains 9 generating buses with 3 HPPs (buses 8, 9 and 10), 6 TPPs (buses 1, 2, 6, 11, 13, 14) and 10 demand buses. Consumer demand functions are given. Cost functions for thermal power plants and limits for power outputs are known for the personnel of this GenCo.

Power plants belong to 8 GenCos, including 5 strategic suppliers and 3 non-strategic suppliers. The network includes 18 ties between the buses.
The scheduling period embraces 3 months: December, January and February. The water volumes accumulated in the HPP reservoirs are known at the beginning and end of the scheduling period.

The scheduling is carried out so that the water spills in each month and for each HPP are equal to zero. This testifies to the rational use of energy resources with implementation of all the water constraints. Results of generation scheduling are presented in Table 1.

As a result, the distorted parameters of cost characteristics (the limits of generation were not distorted in the example) are obtained. They correspond to the optimal solution, which determines the equilibrium of GenCos interests. It is profitable for some producers to distort the parameters of cost functions. Profits of power plants are shown in Table 1 vs. profits obtained after traditional optimization with the total production cost minimization.

The obtained results prove the efficiency of the proposed method and algorithm for medium-term generation scheduling.

\section{CONCLUSIONS}

Generation scheduling is an important problem in the power system operation. The existing scheduling techniques based on total production cost minimization cannot properly reflect the situation in the electricity markets. In the market environment it is reasonable to use a technique based on bi-level and multi-objective

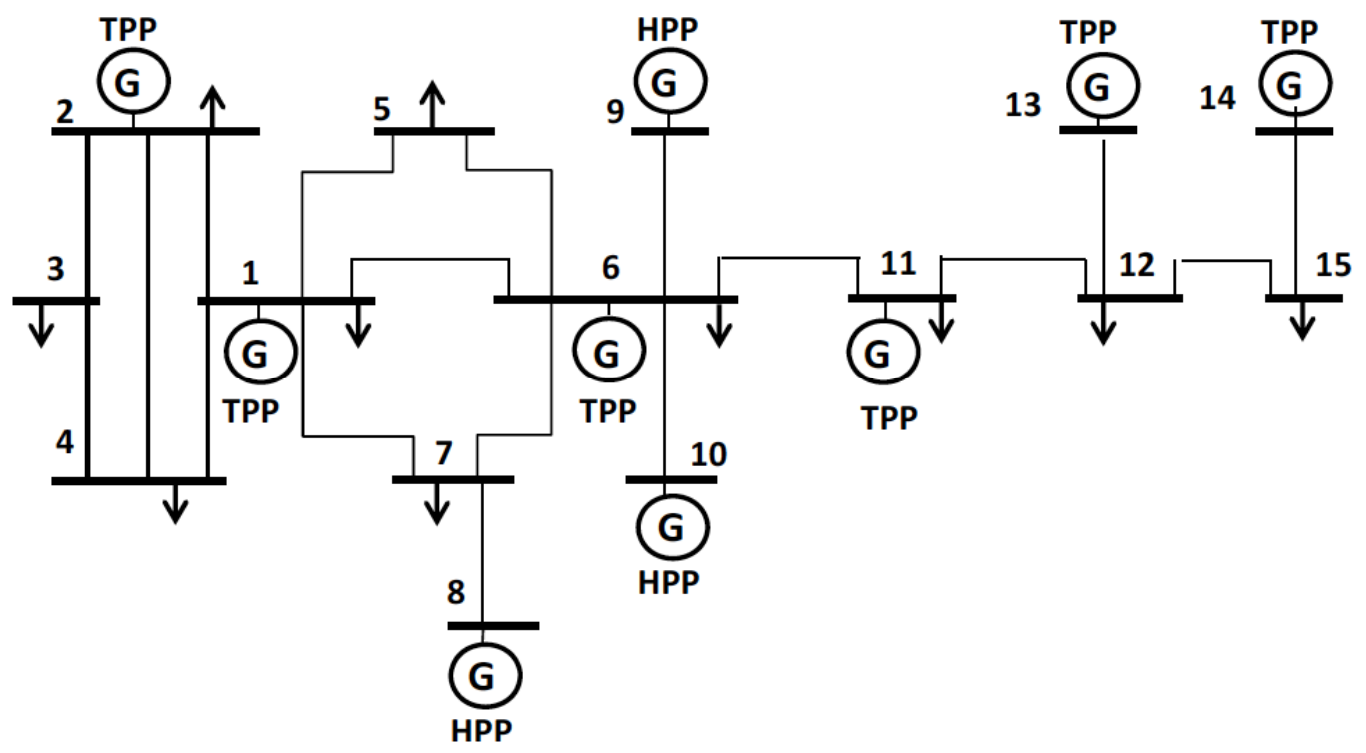

Figure 3: A 15-bus test electric power system. 
Table 1: Scheduling Results

\begin{tabular}{|c|c|c|c|c|c|c|c|c|c|c|}
\hline \multirow{2}{*}{$\begin{array}{l}\text { No. of } \\
\text { bus }\end{array}$} & \multicolumn{3}{|c|}{ Power generation, $M W$} & \multicolumn{3}{|c|}{$\begin{array}{l}\text { Nodal price for generation, } \\
\qquad \$ / M W\end{array}$} & \multicolumn{3}{|c|}{ Distorted parameter ${ }^{*} \bar{b}_{i}$} & \multirow{2}{*}{$\begin{array}{l}\text { Profit of power } \\
\text { plant, thousand } \$^{* *}\end{array}$} \\
\hline & Dec. & Jan. & Feb. & Dec. & Jan. & Feb. & Dec. & Jan. & Feb. & \\
\hline 8 & 5900 & 5900 & 5900 & 15.23 & 15.28 & 15.27 & - & - & - & $14676.6(14162.9)$ \\
\hline 9 & 6700 & 6700 & 6700 & 15.31 & 15.36 & 15.35 & - & - & - & $16754.2(16201.3)$ \\
\hline 13 & 860 & 880 & 875 & 18.2 & 18.26 & 18.25 & $7.9(7.2)$ & $7.9(7.2)$ & $7.9(7.2)$ & $49(48)$ \\
\hline 14 & 400 & 400 & 400 & 18.97 & 19.03 & 19.02 & $13.2(12.1)$ & $13.2(12.1)$ & $13.2(12.1)$ & $760(744.8)$ \\
\hline 1 & 4300 & 4300 & 4300 & 16.6 & 16.65 & 16.64 & $24(24)$ & $24(24)$ & $24(24)$ & $25.3(24.8)$ \\
\hline 6 & 5160 & 5200 & 5200 & 15.83 & 15.88 & 15.87 & $25.1(22.8)$ & $25.1(22.8)$ & $25.1(22.8)$ & $564.2(548.4)$ \\
\hline 10 & 4500 & 4500 & 4500 & 15.61 & 15.66 & 15.65 & - & - & - & $11473.4(11140.7)$ \\
\hline 11 & 3350 & 3400 & 3380 & 15.44 & 15.49 & 15.48 & $22(22)$ & $22(22)$ & $22(22)$ & $221.6(214.7)$ \\
\hline 2 & 2400 & 2400 & 2400 & 14.09 & 14.14 & 14.13 & $15,5(15.5)$ & $15,5(15.5)$ & $15,5(15.5)$ & $160.7(156.5)$ \\
\hline
\end{tabular}

*The true values of parameter $\bar{b}_{i}$ are shown in brackets; ${ }^{\star *}$ The profits of GenCos obtained after the total production cost minimization are shown in brackets.

optimization. A statement of the generation scheduling problem is formulated and presented in the paper.

The bi-level approach allows GenCos to maximize their profits while preparing information about their cost characteristics and limits for generation capacities. The first level sub-problem is solved by each strategic supplier. The sub-problem should be considered as a Nash game with several players.

Generation scheduling in hydrothermal electric power systems requires consideration of constraints, producers' objectives and power outputs for all time intervals over the whole medium-term scheduling period. The method of dynamic programming is applied to generation scheduling.

An algorithm based on the decomposition of the scheduling problem into direct and inverse stages is developed. The algorithm is used to plot the Future Profit Functions for GenCos. The Kuhn-Tucker optimality conditions are introduced in addition to technical constraints to represent the second level subproblem.

Case study demonstrates the behavior of strategic suppliers and efficiency of the suggested method and algorithm.

\section{ACKNOWLEDGEMENT}

The study is supported by the Grant of Russian Leading Scientific School, \# 4711.2014.8

\section{APPENDIX}

\section{Notation}

\section{Indices}

$t=$ index of time interval,

$l=$ index of supposed water volume value,

$m \quad=$ index of water inflow scenario,

$f=$ index of Generation Company,

$I_{n} \quad=$ set of bus indexes in power system,

$I_{g} \quad=$ set of generation bus indexes in power system,

$I_{f} \quad=$ set of generation bus indexes in the $f$-th Generation Company,

$T \quad=$ set of time intervals in the scheduling period,

$L=$ number of supposed water volume values,

$M \quad=$ number of water inflow scenarios.

\section{Constants}

$P_{a i \max }^{t}=$ generation output limit at bus $i$ at time interval $t$,

$a_{i}^{t}, b_{i}^{t}, c_{i}^{t}=$ coefficients of cost function for thermal plant $i$ at time interval $t$. 


\section{Variables}

$P_{g i}^{t} \quad=$ power generation at bus $i$ at time interval $t$,

$\bar{P}_{\text {aimax }}^{t}=$ distorted generation output limit at bus $i$ at time interval $t$,

$\bar{b}_{i}^{t}, \bar{c}_{i}^{t}=$ distorted coefficients of cost function for thermal plant $i$ at time interval $t$,

$p_{i}^{t} \quad=$ nodal electricity price at bus $i$ at time interval $t$,

$S_{f}^{t} \quad=$ profit of $f$-th Generation Company at time interval $t$,

$E S_{f}^{t+1, T}=$ expected future profit of $f$-th Generation Company from beginning of interval $t+1$ to the end of scheduling period,

$V_{i l}^{t} \quad=\quad$-thvolume of water in reservoir of $j$-th hydro power plant at the beginning of interval $t$,

$\tilde{A}_{i l m} \quad=$ random lateral water inflow to the reservoir of $j$-th hydro power plant at l-th water volumevalue according to $m$-th water inflow scenario.

\section{REFERENCES}

[1] Carraretto C. Power plant operation and management in a deregulated market. Energy 2006; 31: 1000-16. http://dx.doi.org/10.1016/j.energy.2005.02.009

[2] Bushnell J. A mixed complementarity model of hydrothermal electricity competition in the western United States. Operations Res 2003; 51: 80-93. http://dx.doi.org/10.1287/opre.51.1.80.12800

[3] Faria E, Barroso LA, Kelman R, Granville S, Pereira MV. Allocation of firm-energy rights among hydro plants: An Aumann-Shapley approach. IEEE Transactions on Power Systems 2009; 24: 541-51. http://dx.doi.org/10.1109/TPWRS.2009.2016376

[4] Kelman R, Barroso LA, Pereira MV. Market power assessment and mitigation in hydrothermal systems. IEEE Transactions on Power Systems 2001; 16: 354-9. http://dx.doi.org/10.1109/59.932268
Villar J, Rudnick H. Hydrothermal market simulator using game theory: Assessment of market power. IEEE Transactions on Power Systems 2003; 18: 91-8. http://dx.doi.org/10.1109/TPWRS.2002.807061

[6] Hobbs BF, Pang JS. Nash-Cournotequilibria in electric power markets with piecewise linear demand functions and joint constraints. Operation Res 2007; 55: 113-27. http://dx.doi.org/10.1287/opre.1060.0342

[7] Ruiz C, Conejo AJ. Pool strategy of a producer with endogenous formation of locational marginal prices. IEEE Transactions on Power Systems 2009; 24: 1855-66. http://dx.doi.org/10.1109/TPWRS.2009.2030378

[8] Weber JD, Overbye TJ. A Two-level optimization problem for analysis of market bidding strategies. Proceedings of the IEEE PES Summer Meeting; 1999: July 20; Edmonton, Canada; 2: 682-7.

[9] Barquin J, Vazquez M. Cournot equilibrium calculation in power networks: An optimization approach with price response computation. IEEE Transactions on Power Systems 2008; 23: 317-26. http://dx.doi.org/10.1109/TPWRS.2008.919198

[10] Dempe S. Annotated bibliography on bi-level programming and mathematical programs with equilibrium constraints. Optimization 2003; 52: 333-59. http://dx.doi.org/10.1080/0233193031000149894

[11] Hobbs BF, Metzler CB, Pang J-S. Strategic gaming analysis for electric power systems: An MPEC approach. IEEE Transactions on Power Systems 2000; 15: 638-45 http://dx.doi.org/10.1109/59.867153

[12] Bompard E, Lu W, Napoli R. Network constraint impacts on the competitive electricity markets under supply-side strategic bidding. IEEE Transactions on Power Systems 2006; 21: 160-70.

http://dx.doi.org/10.1109/TPWRS.2005.857833

[13] Nechaev I, Palamarchuk S. Mid-term generation scheduling on the basis of a bi-level optimization problem. Proceedings of the 12th International Conference on Environment and Electrical Engineering; 2013: May 5-8; Wroclaw, Poland; p. 237-42.

[14] Nechaev I, Palamarchuk S. Midterm hydrothermal generation scheduling using nonlinear dynamic programming. Proceedings of the IEEE PowerTech Conference; 2013: June 16-20; Grenoble, France; paper A5455IN.

[15] Hobbs BF. Linear complementararity models of NashCournot competition in in bilateral and POOLCO power markets. IEEE Transactions on Power Systems 2001; 16: 194-202.

http://dx.doi.org/10.1109/59.918286 